\title{
Infrastructure-free Shipdeck Tracking for Autonomous Landing
}

\author{
Sankalp Arora, Sezal Jain, Sebastian Scherer, Stephen Nuske, Lyle Chamberlain and Sanjiv Singh
}

\begin{abstract}
Shipdeck landing is one of the most challenging tasks for a rotorcraft. Current autonomous rotorcraft use shipdeck mounted transponders to measure the relative pose of the vehicle to the landing pad. This tracking system is not only expensive but renders an unequipped ship unlandable. We address the challenge of tracking shipdeck without additional infrastructure on the deck. We present two methods based on video and lidar that are able to track the shipdeck starting at a considerable distance from the ship. This redundant sensor design enables us to have two independent tracking systems. We show the results of the tracking algorithms in 3 different environments, 1. field testing results on actual helicopter flights, 2. in simulation with a moving shipdeck for lidar based tracking and 3. in laboratory using an occluded and moving scaled model of a landing deck for camera based tracking. The complimentary modalities allow shipdeck tracking under varying conditions.
\end{abstract}

\section{INTRODUCTION}

Take-off and landing on ships is a necessary capability for rotorcraft operating at sea. Missions for rotorcraft on ships are surveillance, transfer of supplies, and ship to shore operations. Manned helicopters rely on the skill of the pilot to track the deck markings and ship, while current autonomous helicopters rely on additional infrastructure on the deck such as radar beacons or GPS systems to track the deck. This additional infrastructure emits radio signals to communicate with the rotorcraft which is undesirable and an autonomous helicopter can only land on instrumented ships. Additionally, the existing technology is expensive and and renders the rotorcraft unrecoverable in case of failure since there is no back up to the main system.

Here we present a solution that does not require any modification to the ship, and can be used to compute the relative position of the rotorcraft with respect to the ship while sensing any obstacles on the deck. Such an approach can be used either to guide an autonomous rotorcraft or to aid a pilot. Our approach uses both a lidar and camera that each can track and localize against the markings on the shipdeck and do so in a redundant and complementary manner.

The lidar based tracking system is a first of its kind that uses both the reflectance and range information from the lidar scans to align against the shipdeck and landing markings. The algorithm takes into consideration the heaving, pitching and rolling that shipdecks would experience in high seas and explicitly accommodates for the resulting warp in the lidar scans. The tracking range of the system is limited by the range of the lidar.

The authors are with The Robotics Institute, Carnegie Mellon University, Pittsburgh, PA 15213, U.S.A., ssingh.cmu.edu

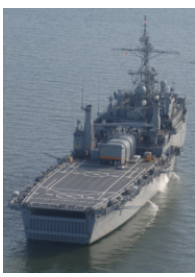

(a)

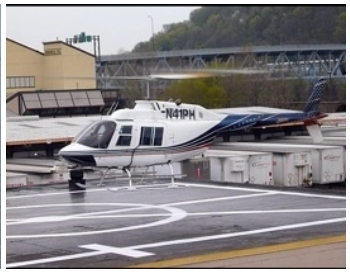

(b)

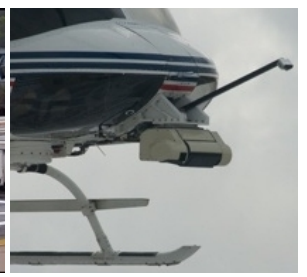

(c)
Fig. 1: (a) Scenario: View of a shipdeck landing pad from a helicopter (b) Test bed: The helipad shown was used to generate data for testing vision and lidar based tracking algorithms. (c) Sensor-head: The lidar and cameras assembled in a single sensor-head unit and mounted on the helicopter. Notice the sensor head is quickly mountable using the same mount-point as used for news cameras.

We also present a camera system that has two purposes: the ability to acquire the shipdeck markings from a long range and also to add redundancy by offering relative position estimates that can be used with the lidar system. Camera and lidar work on two separate illumination sources (sun and laser) resulting in different failure modes and using them together allows the overall tracking system to operate in a wider range of environmental conditions than using just one.

We have put our system through experiments in three different settings; in simulation, in a laboratory with a 1/10th scale shipdeck model actuated to represent ship motion in high-seas and also in flight tests using a helicopter landing on a full-size replica of a shipdeck (See Fig. 1,2).

In this paper we contribute a novel lidar based shipdeck tracking algorithm, results comparing infrastructure-less shipdeck tracking from two different sensor modalities and results for shipdeck tracking in realistic conditions. Detailed analysis of the operation limits of the algorithm is left for the future.

Next we put our approach into context with the state of the art in Sec. II, then explain the lidar and camera tracking algorithms in Sec. III and show our experimental result and setup Sec. IV.

\section{RELATED WORK}

Over the years multiple modalities like relative or augmented global positioning systems (GPS) with data links, radar, infra-red (IR) or visible light cameras and ranging lidar scanners have been suggested for estimating relative pose between rotorcraft and landing decks. Pervan et al. [1] and Gold and Brown [2] suggest a system using a relative 


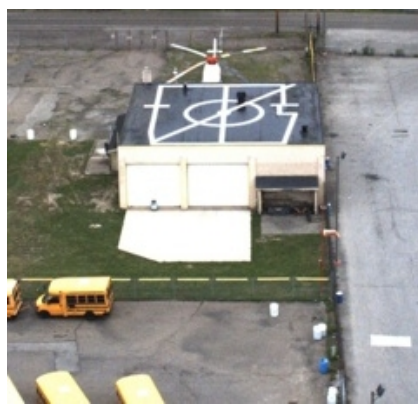

(a) Landing pad visible to camera

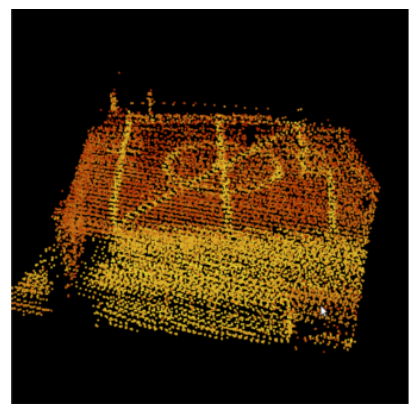

(b) Landing pad point cloud with reflectance values

Fig. 2: Landing Pad (a) The scale and skew of the landing pad as visible to camera changes quickly with distance making tracking a challenging problem. (b) The range and intensity values of the testing landing pad accumulated over time. Information can be gained from both range and reflectance values regarding the location of the shipdeck.

GPS but GPS is subject to availability and is susceptible to jamming. Sierra Nevada Corp developed a RADAR based system called UAS common automatic recovery system (UCARS) [3] for relative pose estimation, it uses a millimeter wave radar on the ship in combination with a transponder on the UAV. It is currently being used by Northrop Grumman Firescout and Bell Eagle Eye. Yakimenko et al. [4] suggest the use of an IR camera to track a ship from long distances using its shape, while relying on GPS with a data link when the shipdeck and rotorcraft are close in. Contrary to this approach $\mathrm{Xu}$ et al. [5] suggest using a GPS/data link for long range localization of the ship and use of an IR radiating $\mathrm{T}$ pattern on the ship deck to facilitate tracking of the ship in close ranges by an IR camera. Xilin et al. [6] propose using a visible light camera and colored beacons on the ship deck to track the landing pad on it. Garrat et al. [7] present a system with a spinning lidar and a single beacon for short distance ship attitude estimation. The lidar data is used in conjunction with the beacon to localize the shipdeck. Saripalli [8] proposes a camera based method to land on moving objects but the motion constraints placed on the object and helicopter make the method unfit for shipdeck tracking.

GPS [1], [2], [4], [5], [6] and radar [3] based methods involve installation of expensive infrastructure on-board the ship and require a data link between the rotorcraft and the shipdeck. The need for infrastructure on the ship renders carrier ships without the required equipment not landable. Same is the case with methods requiring placement of special beacons [6], [7] or patterns [5] on the ship deck. Moreover active sources of radiation like radar, IR or visible light beacons are a risk to the ship in tactical situations. IR or visible light camera based solutions fail in adverse lighting conditions like glare from the sun, fog or rain. The solutions considered do not provide a fall back system if the primary system fails and are designed specifically to facilitate automating landing of a helicopter on a shipdeck.

We address the need for an infrastructure free system for landing deck pose estimation. We present a novel solution which uses complimentary modalities of camera and lidar independently to track the shipdeck. In such a system the camera can track the shipdeck pattern from large distances when the lighting conditions are favorable. In unfavorable lighting the lidar, returning range and reflectance values, can be used to track the shipdeck. Systems to detect and track a landing pattern have been developed before [9], our particular approach is different in that it can be adapted to any particular pattern because the image and lidar based algorithms are generic in nature, just needing a template as input. The presented solution facilitates autonomous operation on land with visual and range sensing providing with the necessary data for finding [8] and evaluating landing sites [10] while avoiding obstacles.

\section{APPROACH}

\section{A. Lidar Based Tracking}

The need for tracking the shipdeck pose in varying light conditions, while requiring no infrastructure on the deck makes lidar an obvious choice as a perception modality. Lidar, unlike a camera, does not give information of the whole shipdeck at once and due to the unknown motion of the shipdeck the recorded point cloud is warped in space. To overcome this problem and to maintain a high update rate while tracking, we cannot save the point cloud over time and process it in a batch manner, rather we have to use the range/reflectance data as and when it arrives. However, the instantaneous data using the points reported in a single scan lacks sufficient information to localize the ship deck. Since the data is dependent on the structure of the environment, which is the discontinuous surface of a moving shipdeck, it is not trivial to find a parametric solution of the observation model. A particle filter based solution can overcome both these issues as it tracks the particles over time and has no parametric form of distribution attached to it. The search space for a ship deck tracker is the 6-dof deck pose (Eq.1).

$$
\vec{x}=\left[x_{\text {ship }}, y_{\text {ship }}, z_{\text {ship }}, \phi_{\text {ship }}, \theta_{\text {ship }}, \psi_{\text {ship }}\right]^{T}
$$

where $x_{\text {ship }}, y_{\text {ship }}, z_{\text {ship }}, \phi_{\text {ship }}, \theta_{\text {ship }}, \psi_{\text {ship }}$ are the position and orientation of the ship respectively

A particle filter in 6 dimensions will require a large number of particles, which is prohibitive in running the tracker in real time especially when using lidar data requires the expected observation, for each particle, to be calculated by using raycasting. We overcome this problem by exploiting the constraint that the landing deck is planar and we can compute the $\phi, \theta$ and $\mathrm{z}$ of the deck if we know the location of ship in the $x-y$ plane and its yaw angle. Hence we find a plane fit on the warped lidar data (Section III-A.2.a) and use the information from the normal to support the particle filter and reduce its search space to 3 dimensions (SectionIII-A.2) given by $\mathbf{x}, \mathrm{y}$, and $\psi$ ( Fig. 3 ). It is important to remember 
here that even though the search space of the particle filter is 3 dimensional the trackers output is full 6 DOF state of the ship. We use a robust sum of exponentiated distances (SED) based observation model (Section III-A.1) to update the weights of the particles using observed reflectance $\left(i_{o}\right)$ and range $\left(r_{o}\right)$ readings.

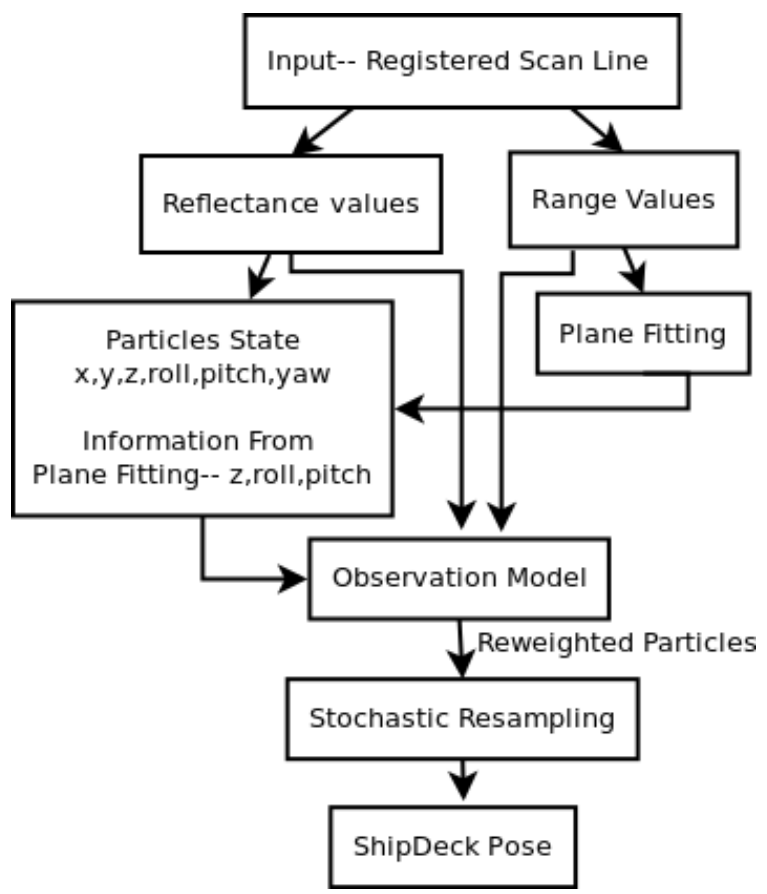

Fig. 3: Particle Filter Block Diagram. The lidar outputs range and reflectance values form input to the tracker, range values are used for RANSAC [11] based plane fitting on a rolling buffer of points, while both range and reflectance values are used in the observation model. Plane fitting provides the $\mathrm{z}$, $\phi, \theta$ for an expected $\mathrm{x}, \mathrm{y}$ and $\psi$ of the ship deck. This setup reduces the dimensionality of the problem, hence requiring less number of particles, making the algorithm efficient.

1) Observation Model: The range reading in a single scan of horizontal FOV by the lidar provide ambiguous information regarding the location of the ship as multiple ship locations can result in similar range values in a scan. To partially disambiguate the solution and to make the filter converge at a higher rate we exploit reflectance information from the lidar.

For each incoming scan line, the maximum range readings are discarded as they provide no information and add processing overhead. Direction vectors of the non-maximal range readings are used to calculate the expected range $\left(r_{e}\right)$ and reflectance $\left(i_{e}\right)$ readings for each particle. Every particle suggests a different location of the shipdeck model, making it necessary to repeat raycasting for each particle. Therefore, for each particle we have $m$ range and reflectance values, if $m$ is the number of non-maximal range returns from the scanner.

A single scan line of range/reflectance data can vary drastically even when there is a small difference in the position of particles in the state space. However, we need a smooth observation model that changes gradually with change in the position of the particles, such that even with fewer particles the particle filter can gradually converge towards the correct solution. Standard one dimensional distances like Minkowski distance or correlation are discontinous for the input data hence we design exponentiated distances (SED) based observation model in range and reflectance values.

$$
\begin{gathered}
d\left(\vec{r}_{e}, \vec{r}_{o}\right)_{\text {range }}=\sum_{j=1}^{m} 1-\exp \left(-a b s\left(r_{e}^{j}-r_{o}^{j}\right) / \rho_{\text {range }}\right) \\
d\left(\vec{i}_{e}, \vec{i}_{o}\right)_{\text {intensity }}=\sum_{j=1}^{m} 1-\exp \left(-a b s\left(i_{e}^{j}-i_{o}^{j}\right) / \rho_{\text {intensity }}\right) \\
w_{p}=e^{-d\left(\vec{r}_{e}, \vec{r}_{o}\right)_{\text {range }} / \varrho_{\text {range }} * e^{-d\left(\vec{i}_{e}, \vec{i}_{o}\right)_{\text {intensity }} / \varrho_{\text {intesity }}}} \text { (4) }
\end{gathered}
$$
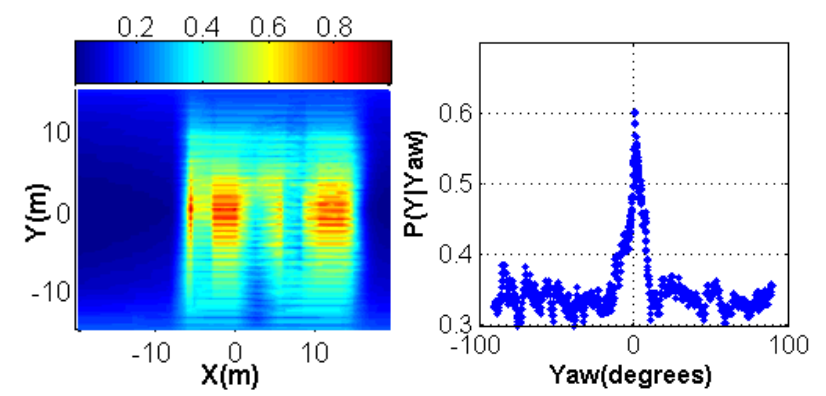

(a) $\mathrm{P}(\mathrm{Y} \mid \mathrm{x}, \mathrm{y})$ for the correct yaw.(b) $\mathrm{P}(\mathrm{Y} \mid \mathrm{yaw})$ for the correct $\mathrm{x}$ and $\mathrm{y}$. The ground truth is at $(0,0)$. The ground truth is at $0^{\circ}$

Fig. 4: Spatial smoothness of Observation Model (a) \& (b) Demonstrate the smoothness of the observation model in $\mathrm{x}, \mathrm{y}$, yaw. Notice how the observation model values rise gradually towards the ground truth. This implies the density of particles required to converge at the correct solution is low

As can be seen in (Fig.4) the observation model rises gradually in value as it reaches the ground truth. This implies that even if the trackers belief is away from the ground truth it is likely to converge to the correct position with time. The smoothness of the observation model can be attributed to the function $1-e^{-x}$ that maps $R^{1}$ to $[0,1)$. Hence, large differences in expected range/intensity to observed range/intensity values contribute a small amount to the total distance. Further the behavior of the observation model can be controlled by tuning the parameters $\rho_{\text {range }}, \rho_{\text {intensity }}, \varrho_{\text {range }}, \varrho_{\text {intensity }}$. One of the interesting observations in Fig.4a is the presence of two maxima. These are present due to the fact that shipdeck landing markings are symmetrical and range values do not vary much when they lie on different parts of a rectangular deck. However as the lidar point moves up and down on the deck the ambiguity is resolved with more incoming information. 
2) Search space reduction and plane fitting: This section covers the techniques and assumptions we use to reduce the search space of the particle filter based tracker. If we know the equation of the ship deck plane (Eq.5), we can compute the height, roll and pitch of the ship if the heading/yaw and the location of the landing pad on the xy plane are known (Eq. 6, 8, 9). These three dimensions constitute the search space for our filter. To extract the landing deck plane, we assume the biggest planar surface observed closest to the horizontal is the shipdeck plane. The following section describes the plane fitting algorithm used to extract a plane from a warped point cloud.

$$
\begin{gathered}
a x+b y+c z+d=0 \\
z_{\text {ship }}=-d / c\left(a x_{\text {ship }}+b y_{\text {ship }}\right) \\
\hat{n}=[a, b, c] / \sqrt{a^{2}+b^{2}+c^{2}} \\
\theta_{\text {ship }}=\operatorname{atan} 2\left(\hat{n}_{x} \cos \phi_{\text {ship }}+\hat{n}_{y} \sin \phi_{\text {ship }}, \hat{n}_{z}\right) \\
\psi_{\text {ship }}=\operatorname{atan} 2\left(\hat{n}_{x} \sin \phi_{\text {ship }}-\hat{n}_{y} \cos \phi_{\text {ship }}, \hat{n}_{z} \cos \phi_{\text {ship }}\right)
\end{gathered}
$$
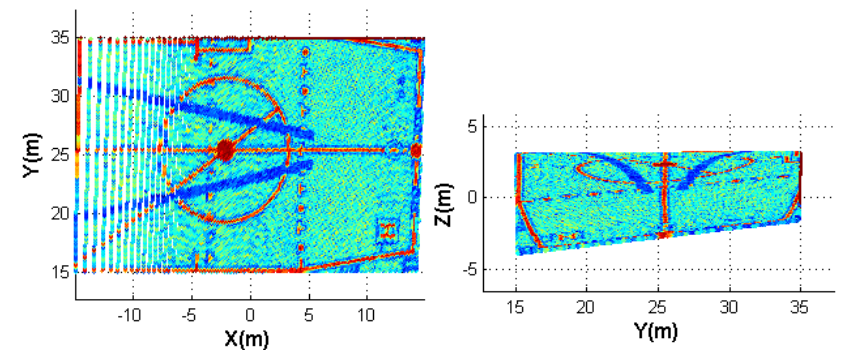

(a) Top view of a warped point cloud(b) Front view of a warped point of ship deck under motion cloud of ship deck under motion

Fig. 5: Warped Shipdeck. Top and front view of simulated point cloud of a ship deck registered without compensating for motion. A plane fit to this point cloud leads to a poor fit and erroneous results, hence the need for a fitting a plane on a small temporal rolling buffer of the point cloud.

a) Plane Fitting: The point cloud of a planar region like the landing pad looks warped due to the unknown motion of ship deck (Fig. 5). Therefore, to fit a plane we apply a RANSAC based plane fitting on a temporal rolling buffer of the point cloud. It is important to pick the correct size of the rolling buffer, a too small buffer will lead to a bad plane fit while in case of too big, the data is highly non-planar due to warp. Through experimentation we found that a rolling buffer of 0.1 seconds produces satisfactory results for $20 \%$ s change in normal of the plane when the laser nodding rate is $60 \%$ s. Once all the planes are extracted, planes with normals more than $7^{\circ}$ degrees away from the previous segmented normal are rejected. We assume the shipdeck normal will vary at a maximum rate of $70 \%$ s. Then we pick the biggest plane, closest to the horizontal as the shipdeck plane.
3) Propagation Model: Shipdeck motion might seem periodic but due to wave slaps and unknown ocean conditions, the variation in the shipdeck motion can be unpredictable. Therefore, we do not make any assumption about the motion of the shipdeck and the particles are randomly propagated using a normal distribution through the state space.

4) Lidar Directional Control: The vertical field of view of the lidar is actively controlled to keep just the landing pad in focus. It is necessary to keep the ship localized, since the motion parameters of the shipdeck are not estimated and if the lidar looses the sight of the shipdeck for too long the tracking algorithm will diverge. Focusing of the lidar on the landing site ensures that the landing deck is almost always in view, hence the plane segmentation can update its belief of the shipdeck plane at a higher rate. Since the tracker's belief of the shipdeck can be erroneous, the vertical FOV of the lidar is kept more than the angle subtended by the deck on the lidar. The FOV is chosen such that the lidar beams still fall on the deck in the case of a position error of $+/-20 \mathrm{~m}$

\section{B. Camera Based Tracking}

We have developed a camera-based approach that first finds the landing pattern on the shipdeck, dealing with initialization from an unknown pose, and then moves into a tracking phase that operates in real-time and can deal with the significant scale change of the landing pattern in the images. The camera-based tracking system serves multipule purposes- it adds redundancy, enables the landing pattern on the shipdeck to be acquired from a long range, and it provides input pose to initialize the lidar tracking system.

The input to the algorithm is a template image of the landing pattern, and a set of points manually marked both in ship and image coordinates to give the system metric scale. The template is masked to confine the shipdeck region to restrict the image matching. The algorithm is initialized using SIFT feature matches [12] between the template and the observed image. We assume the landing pattern is within the field of view at the time of initialization. Since the template image can be from a significantly different viewpoint than the observed image, we use image-warping A-SIFT approach [13] to ensure that sufficient matches are found. A homography transform is fit to the matched points, using RANSAC, and used to find the location of the template points from the initial template transformed to the current image. We now have a set of points in our current input image from the camera and their respective 3D position in the shipdeck frame, robust planar pose algorithm is used [14] to translate the homography to give a single solution of the 6DOF pose of the rotorcraft in the shipdeck frame.

After an initial calculation of the homography using SIFT, the shipdeck template is tracked from frame to frame using the Lucas-Kanade (LK) algorithm [15]. The homography transforms are accumulated over the frames to give the transformation between the initial template and the input image at any given time, with the robust planar pose estimation repeated to continually update the pose estimate of the rotorcraft. 
1) Recovery: Due to illumination changes or large scale change between consecutive frames or jerks in rotorcraft motion, LK tracking might produce an erroneous result. We assess the calculated motion of the rotorcraft and if it is outside the bounds of dynamic feasibility for the robot, a recovery procedure is followed. This recovery procedure reinitializes the system with respect to the template image using the SIFT-based initialization procedure.

2) Adaptive-template : As the rotorcraft is moving directly towards the landing pattern from a distance $>300 \mathrm{~m}$ to finally land on it, one of the major problem in tracking is the scale change of the shipdeck from the template to the current image. The recovery algorithm based on SIFT is efficient but does not work beyond a range of scale differences. To adapt to this, the template itself is updated every three seconds and the homography relating this template to the original template is stored. Whenever the LK tracking system fails, the updated template can be used for the recovery procedure to re-initialize and its relationship to the original template is applied to find the pose of rotorcraft. The error in the pose of updated template accumulates over time. This can be prevented by using several manually marked templates at varying distances from the shipdeck.

3) Final-approach : When the rotorcraft is nearing the landing pattern (at about 30-40 $\mathrm{m}$ away from the landing pattern), parts of the pattern starts moving out of the fieldof-view of the forward facing camera. To localize in the final approach phase, we use a downward facing camera that always keeps the target in the field of view. Tracking through the downward facing camera operates in the same way as the forward facing camera but with a different manually set initial template. The different initial template for the downward facing camera serves two purposes. First it is easier to track as we select an image with a perspective and scale similar to that seen in the downward camera during the final stages and it also defines the template points in a much higher resolution, to achieve a greater accuracy in pose estimation - a requirement for close-in precision control.

\section{Sensor Design}

Tracking the shipdeck using cameras and lidar required us to design a sensor which is capable of tracking the landing pattern for varying distances using multiple modalities while keeping track of the pose of the vehicle. The sensor suite is designed such that lidar can be used to evaluate landing sites on land, as well as actively keep track of the ship in sea. We built an integrated sensor suite (Fig. 6) with a scanning lidar range finder, one forward facing highresolution camera and two downward facing cameras. It worked reliably while deployed on a manually operated Bell 206 for the deck tracking experiments. Data from cameras and lidar scanner was recorded in sync with RTK GPS data for offline processing. Sec.III-D and Sec.III-E provide further details on the components and design considerations of the sensor suite.

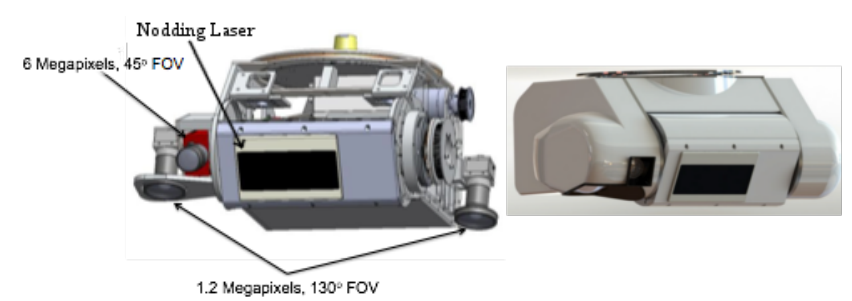

(a) CAD rendering of open sensor suite (b) actual encased sensor suite

Fig. 6: Sensor Suite: Dimensions: 17.5" x 14.2" x 8", Weight: $30 \mathrm{lbs}$

\section{Camera Suite Design}

We use a two camera system, one a forward facing highresolution cameras fitted with $16 \mathrm{~mm}$ lens and we use a uEye with fish-eye -1 lens in a downward facing configuration, as seen in Fig. 6a. The forward and downward facing cameras are oriented with enough overlap to transition from tracking the landing pattern in the forward camera to the downward camera, as the rotorcraft approaches the shipdeck, see Fig. 7. As a plan for future development, we have also included a second downward facing camera in the design to incorporate a stereo visual odometry algorithm, although for results in this paper we use just the left of the two downward facing cameras.

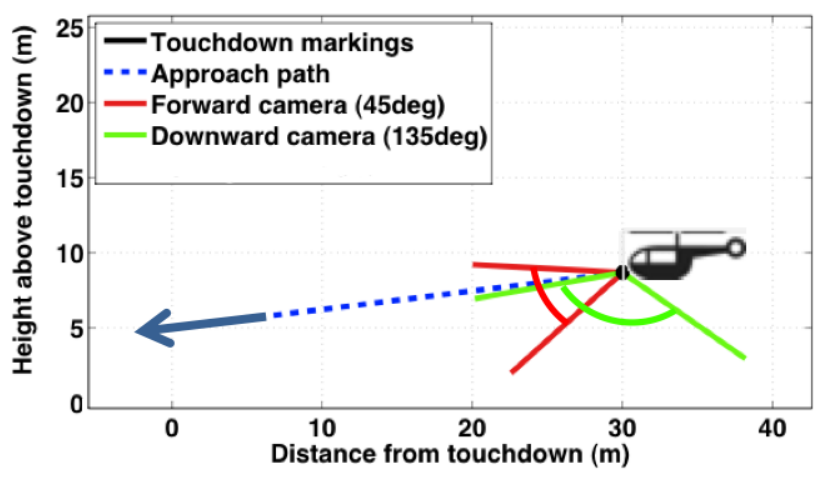

Fig. 7: Camera Configuration: Graph visualizing the fieldof-views of the front facing and downward facing camera.

\section{E. Lidar Design}

The lidar is required to return both range and reflectance values while being able to operate in changing light conditions. It should be able to cover the changing field of view of the ship deck with as few moving parts as possible. We built a nodding lidar system with 180 degrees vertical nodding field of view (FOV), 85 degrees horizontal FOV and a 34 $\mathrm{kHz}$ measurement rate. We used a $100 \mathrm{~m}$ range sensor with 4 beams. The nodding motion profile of the lidar was chosen so that it can be actively pointed downwards, for a high density of points on the landing zone and at an angle to point towards the ship deck to keep its track. The pointing 
angle of the lidar and its nodding speed between the start and end angles is controllable.

\section{Results}

The camera and lidar based tracking algorithms were tested on data collected from flight runs on a helicopter. The lidar based algorithm was also tested in simulation with a moving ship. The camera based tracking algorithm was also tested in laboratory on scaled model of the shipdeck.

\section{A. Simulation}

The simulation environment gives us the facility to test the lidar based tracking method with a moving shipdeck. The ship model used is a mesh with textures on the faces used for reflectance value mapping. The reflectance values are assumed to be invariant with incident/reflectance angle. We use a naive shipdeck motion model with the roll, pitch and yaw of the ship varying sinusoidally. We provide initial results for simulation data in Fig 8.
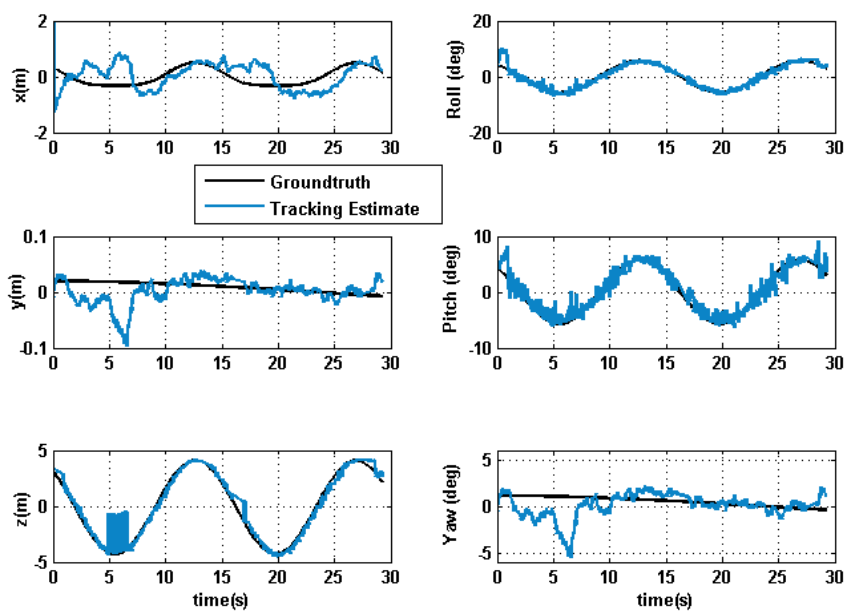

Fig. 8: Simulation results. Estimated shipdeck pose and ground truth in 6-dof for a moving shipdeck by lidar based tracker. The co-ordinate system origin is at the center of the shipdeck at the time of start of the experiment.

The simulation is run with the ship normal changing at a maximum rate of $7 \%$. This results in an average rate of change of height of shipdeck to be 8 meters for every 10 seconds. We use the pose data recorded from test runs for helicopter approaches in our simulation (Fig. 11a). The moving shipdeck is placed 2 meters below the end point of the helicopter trajectory. The helicopter approaches the shipdeck with a glide slope of almost $14^{\circ}$ and starts at a distance of 120 meters from it. The tracker is initialized 102 meters away from the shipdeck position. The algorithm quickly converges to the correct solution and keeps track of the shipdeck pose using just 100 particles. The particle filter recovers from the large initialization error $(20 \mathrm{~m})$ due to the smoothness and continuity of the observation model and reduction of state space into 3 dimensions. The average error in the estimated position of the shipdeck after convergence
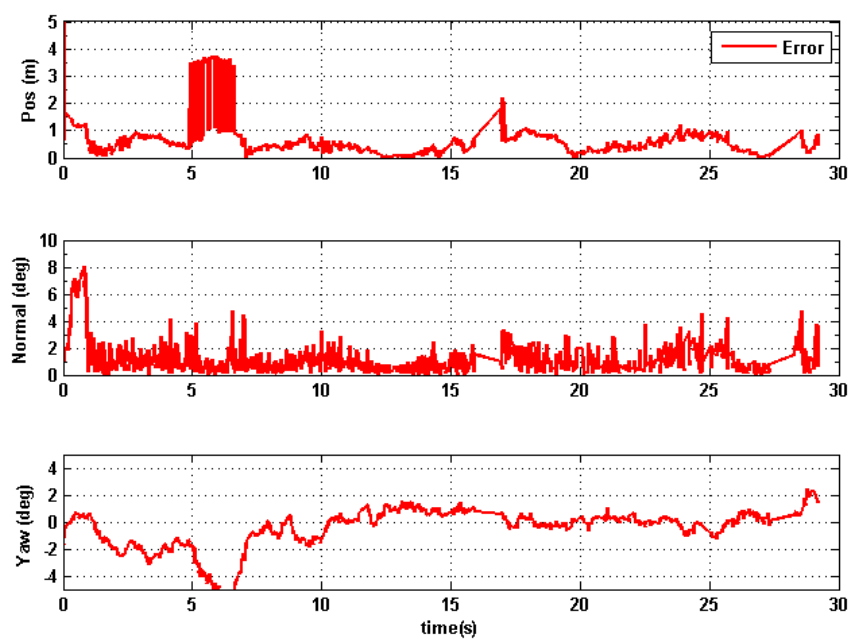

Fig. 9: Tracking Error. Error in shipdeck tracking with respect to time between the helicopter and the landing pad using lidar based tracking. The axis in the graphs do not show maximum error to display sufficient resolution at low errors.

of the particle filter is 0.3581 meters, while the average orientation error is $0.9524^{\circ}$. The divergence of the tracker around the 5 second mark is due to the plane fitting algorithm detecting multiple planes on the ship that could be considered as the landing pad. We can get rid of multiple shipdeck candidates by choosing the plane closest to the last tracked location, but it leads to the tracker not recovering from tracking the wrong plane.

\section{B. Laboratory}

To demonstrate the ability of the camera based tracker system on real sensor data we present, a mock-shipdeck setup in our laboratory with a 1/10th scaled down foamcore model with the helicopter landing pattern painted to scale. The shipdeck model was actuated to represent ship motion in high-seas with roll and pitch rates of 25 degrees per second. As a stark illustration of the success of the tracking system, we present the lidar scans of the shipdeck without any registration to the moving ship coordinate frame and compare with the lidar scans registered using the tracked estimate of the relative pose between sensors and shipdeck, see Fig. 10.

\section{Field Experiments}

The sensor suite with data logger was mounted on a Bell 206 helicopter. The helicopter was operated manually to approach and hover a few centimeters over the landing pattern modeled after standard US Naval ship deck markings for rotorcraft. The landing approach profile of the helicopter was similar to those of rotorcrafts landing on shipdecks (Fig.11a). The time synced image, lidar and GPS/INS data collected during the runs was then given as input to the camera and lidar based tracking algorithms. 


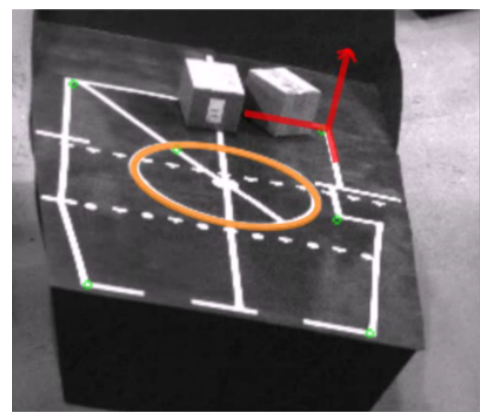

(a)
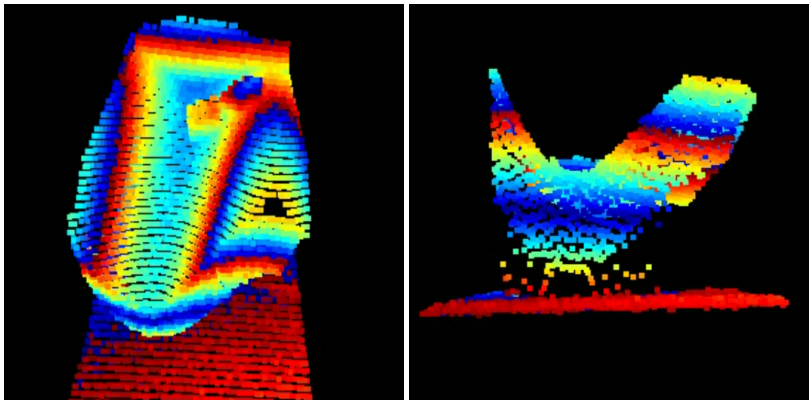

(b)
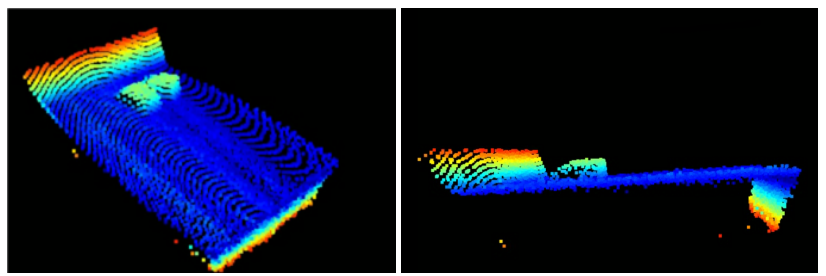

(c)

Fig. 10: Laboratory Results (a) Image of the laboratory tests with a 1/10th scaled ship model (approximately $2 \mathrm{~m}$ wide by $4 \mathrm{~m}$ long). The ship was moved to represent motions in high seas, with roll and pitch rates of 25 degrees. Colored markings are displayed to show the visual tracking estimate. (b) Visualization of the lidar scans that are not registered into the shipdeck coordinate frame (left: overhead view of shipdeck, right: side-view of shipdeck). The shipdeck is moving whilst the lidar is performing the sweeping scan, and as a result, the shipdeck is warped in the lidar scans. (c) lidar scans registered using the tracking system (Bottomleft: overhead view of shipdeck, Bottom-right: side-view of shipdeck). Here the accurate tracking systems, enables the lidar scans to be registered correctly and the shipdeck is estimated as a single flat surface. Scans are colored by height. In addition the obstaces on the surface can also be detected.

Fig. 11 confirms that the algorithms track the landing pattern successfully. An initial error of $6 \mathrm{~m}$ is artificially induced in case of lidar based tracker by initializing the particle filter away from the ground truth. Due to high variance the tracker's belief initially jumps but as the lidar readings become more informative the belief of the tracker starts converging towards the ground truth. The mean error in position after the tracker converges is $0.311 \mathrm{~m}$. When the
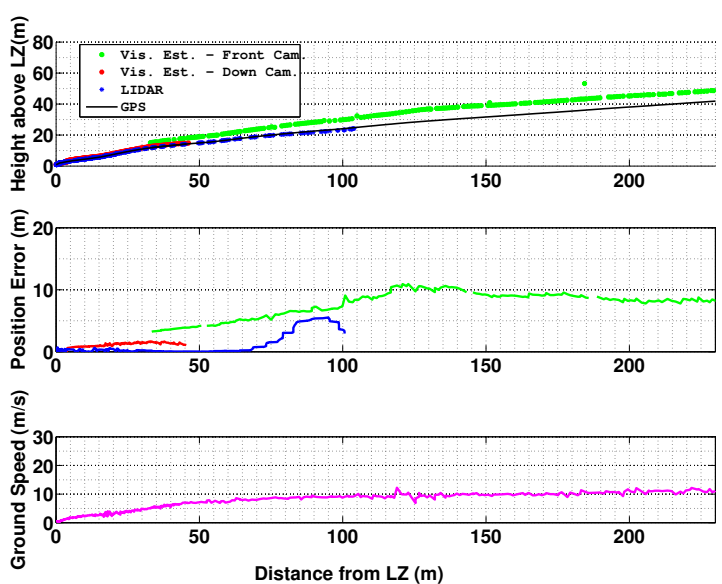

(a) Height, Position error and speed profile as observed during tracking

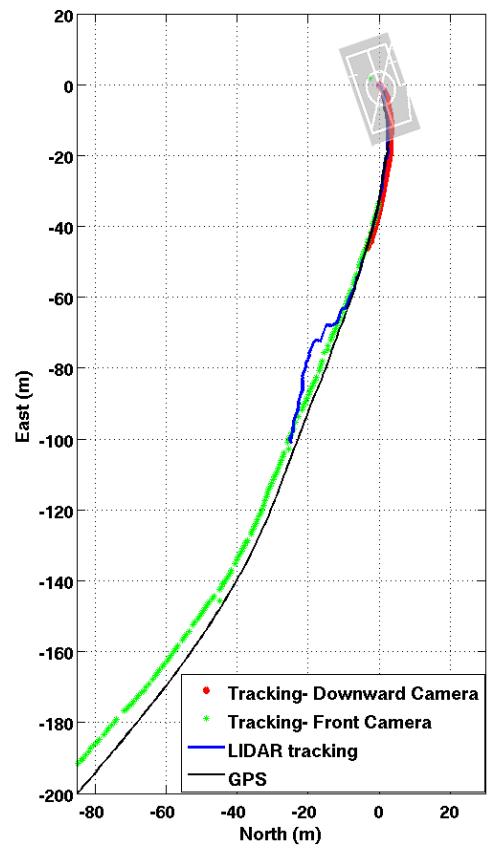

(b) Lidar and Visual Tracking - Top-Down view

Fig. 11: Field test results. Comparison of Error plots and trajectory of the vehicle estimated by lidar and visual tracking system against GPS ground-truth.

helicopter reaches close to the the landing pad, the lidar FOV does not cover the whole pad and the information regarding the edges of the landing pad is no longer present. Hence the error in the tracker increases by a small amount. The information given by intensity values is sufficient for keeping the tracker localized.

We present sample images of the visual system in Fig.12. The landing pattern is acquired at a long range when it forms a small area in the image. It is tracked all the way with a significant scale change until it begins to leave the bottom edge of forward camera's view and we hand the tracking to the wide field-of-view downward camera. 


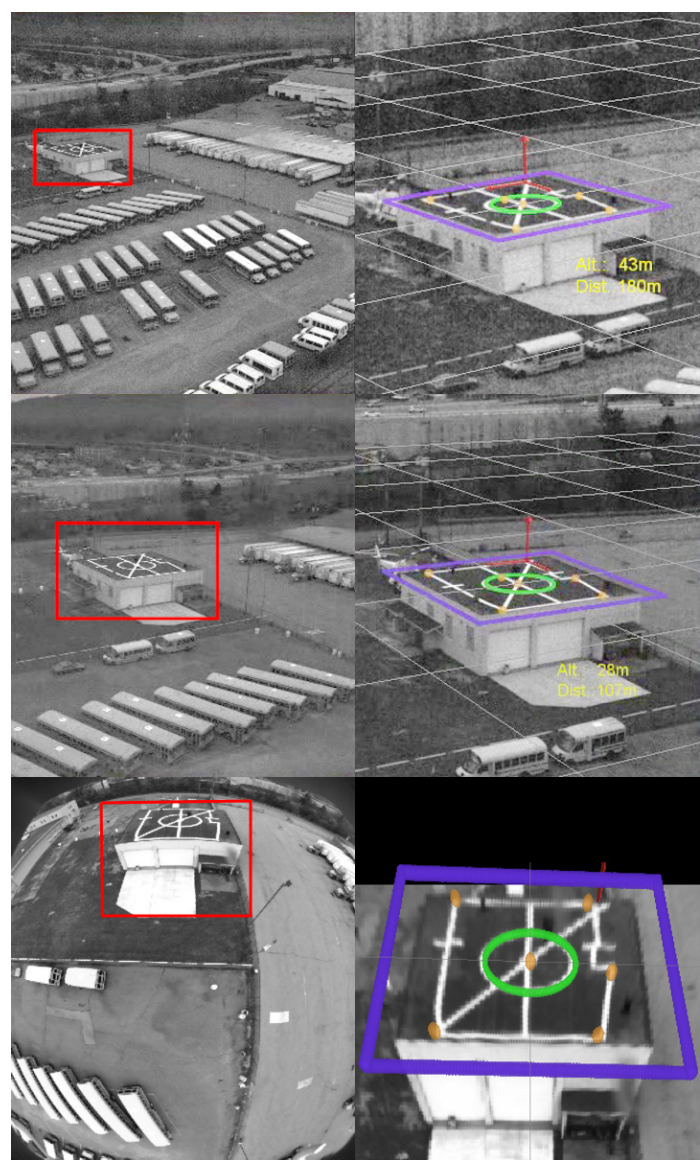

Fig. 12: Visual tracking of shipdeck landing pattern during approach. Left: Raw images, with red-rectangle drawn from distant images to highlight position of landing pattern. Right: Zoomed images with landing markings visualized on top to illustrate state of visual tracking algorithm. Top: forward camera whilst rotorcraft is at $180 \mathrm{~m}$ from landing pattern, middle: forward camera whilst rotorcraft is at $100 \mathrm{~m}$ from landing pattern, bottom: downward camera whilst rotorcraft is at $30 \mathrm{~m}$ from landing pattern.

\section{CONCLUSION}

In this paper we described a shipdeck tracking system which requires no special infrastructure on the ship. As a part of the system we presented a novel lidar based tracking algorithm and a vision based shipdeck tracker. The vision based system was successfully put through a variety of tests, including tracking, heaving, pitching and rolling shipdeck motions much larger than those expected in rough seas, also we have tested with obstacles occluding the landing pattern, showing the algorithm can handle such disturbances. The lidar based system was also tested in simulation for a moving ship deck. In flight tests we evaluated both the lidar and camera based methods with a full size replica of a ship deck, albeit static, without any substantial occlusion of the landing pattern. One important lessons learnt is that, at long ranges, a small change in the laser pointing angle can result in laser completely missing the shipdeck. The laser direction controller should be designed such that it is tolerant to errors in tracking pose.

In future, we intend to deploy in flight tests on a real ship with typical clutter that would be present on the deck and evaluate the system in conditions like night, fog, rain and sun glare etc. to test its feasibility and operating limits. We believe the system can handle varying environmental conditions owing to the redundancy in modalities used and detect obstacles on the deck using the lidar sensor.

\section{ACKNOWLEDGMENT}

The authors gratefully acknowledge Sanjiban Choudhury's help with system development and experimentation. We would also like to thank our pilot Dan Sweazen for safe and productive flights.

\section{REFERENCES}

[1] B. Pervan, F. cheng Chan, and G. Colby, "Performance analysis of carrier-phase dgps navigation for shipboard landing of aircraft," NAVIGATION, pp. 181-191, 2003.

[2] K. Gold and A. Brown, "A hybrid integrity solution for precision landing and guidance," in Position Location and Navigation Symposium, 2004. PLANS 2004, april 2004, pp. 165 - 174.

[3] S. N. Corporation, "UCARS-V2 UAS common automatic recovery system - version2," 2008. [Online]. Available: http://www.sncorp. com/pdfs/cns_atm/UCARS-V2ProductSheet.pdf

[4] O. A. Yakimenko, I. I. Kaminer, W. J. Lentz, and P. A. Ghyzel, "Unmanned aircraft navigation for shipboard landing using infrared vision," IEEE Transactions on Aerospace Electronic Systems, vol. 38, pp. 1181-1200, Oct. 2002.

[5] G. Xu, Y. Zhang, S. Ji, Y. Cheng, and Y. Tian, "Research on computer vision-based for uav autonomous landing on a ship," Pattern Recogn. Lett., vol. 30, no. 6, pp. 600-605, Apr. 2009.

[6] X. Yang, L. Mejias, and M. Garratt, "Multi-sensor data fusion for uav navigation during landing operations," in Australasian Conference on Robotics and Automation (ACRA 2011). Monash University, Melbourne, VIC: Australian Robotics and Automation Association Inc.,Monash University, 2011, pp. 1-10.

[7] M. Garratt, H. Pota, A. Lambert, S. Eckersley-Maslin, and C. Farabet, "Visual tracking and lidar relative positioning for automated launch and recovery of an unmanned rotorcraft from ships at sea," Naval Engineers Journal, vol. 121, no. 2, pp. 99-110, 2009.

[8] S. Saripalli, "Vision-based autonomous landing of an helicopter on a moving target," Proceedings of AIAA Guidance, Navigation, and Control Conference, Chicago, USA, 2009.

[9] F. Kendoul, "Survey of advances in guidance, navigation, and control of unmanned rotorcraft systems," J. Field Robotics, vol. 29, no. 2, pp. 315-378, 2012.

[10] S. Scherer, L. Chamberlain, and S. Singh, "Online Assessment of Landing Sites," in AIAA Infotech@Aerospace, Atlanta, Apr. 2010.

[11] M. A. Fischler and R. C. Bolles, "Random sample consensus: a paradigm for model fitting with applications to image analysis and automated cartography," Commun. ACM, vol. 24, no. 6, pp. 381-395, Jun. 1981.

[12] D. G. Lowe, "Distinctive image features from scale-invariant keypoints," Int. J. Comput. Vision, vol. 60, no. 2, pp. 91-110, Nov. 2004.

[13] J.-M. Morel and G. Yu, "Asift: A new framework for fully affine invariant image comparison," SIAM J. Img. Sci., vol. 2, no. 2, pp. 438-469, Apr. 2009.

[14] G. Schweighofer and A. Pinz, "Robust pose estimation from a planar target," IEEE Transactions on Pattern Analysis and Machine Intelligence, vol. 28, pp. 2024-2030, 2006.

[15] S. Baker and I. Matthews, "Lucas-kanade 20 years on: A unifying framework," Int. J. Comput. Vision, vol. 56, no. 3, pp. 221-255, Feb. 2004 